\title{
Free Vibration Analysis of Embedded Swcnts using Dqm Based on Nonlocal Euler-bernoulli Beam Theory
}

\author{
Maria Anna De Rosa ${ }^{1}$, Maria Lippiello ${ }^{2}$, Hector Martin ${ }^{3}$, Francesco Vairo ${ }^{1}$ \\ ${ }^{1}$ School of Engineering, University of Basilicata, Potenza 85100, Italy \\ ${ }^{2}$ Department of Structures for Engineering and Architecture, University of Naples "Federico II", Naples 80134, Italy \\ ${ }^{3}$ Regional Academica Reconquista, Universidad Tecnologica Nacional, Reconquista, Santa Fe 3560, Argentina \\ maria.derosa@unibas.it,maria.lippiello@unina.it, hdmartin@criba.edu.ar
}

\begin{abstract}
The present paper deals with the free vibration analysis of single-walled carbon nanotube in an elastic medium based on nonlocal Euler-Bernoulli beam theory. The differential quadrature method (DQM) is applied to finding free frequencies of nano-beam systems having to the ends translational and rotational elastic constraints and taking into account the influence of the rotary inertia. Numerical examples are performed to show the accuracy of the proposed method. DQM.

Index Terms - carbon nanotube, free vibration, nonlocal effect,
\end{abstract}

\section{Introduction}

Carbon nanotubes (CNTs) constitute a prominent example of nanomaterials and nanostructures, which have attracted attention by many researches due to their extraordinary electrical, physical and mechanical properties. Extensive studies have been conducted on studying the mechanical properties of CNTs such as bending, buckling and post buckling [1-2] and free vibration [3]. Recently, many nonlocal elastic continuum models have been used for studying the mechanical behaviour of CNTs including beam models. Their application to the analysis of CNTs allows to evaluate of the small-scale effects influence, [4-5].

The present paper studies free vibration of embedded SWCNT based on nonlocal Euler-Bernoulli beam theory. The surrounding elastic medium is described as the Winkler and Pasternak models, defined by the $k_{\mathrm{w}}$ and $k_{\mathrm{p}}$ costants. The Hamilton's principle is applied to derive the governing equations and boundary conditions, which are solved by using the well-known differential quadrature method (DQM), [6-13]. The influences of the elastic medium coefficients, nonlocal parameter and end supports on the free vibrations characteristics of the SWCNT are described.

\section{II . Problem formulation}

Let us consider a single-walled carbon nanotube with length $L$, Young modulus $E$, second moment of area $I$, mass density $\rho$ and cross-sectional area $A$. The nanotube analyzed is assumed to be embedded in an elastic medium and it has to the ends translational $k_{\mathrm{TL}}, k_{\mathrm{TR}}$ and rotational $k_{\mathrm{RL}}, k_{\mathrm{RR}}$ elastic constraints. The elastic media is simulated using the Winkler and Pasternak foundation models.

According to the nonlocal Euler-Bernoulli beam theory and taking into account of the nonlocal effects and the influence of rotary inertia, the equation of motion, for an embedded SWCNTs, is:

$$
\begin{aligned}
& \left(\mathrm{EI}+\eta^{2} \mathrm{k}_{\mathrm{p}}\right) \mathrm{v}^{\prime \prime \prime}(z, \mathrm{t})+\eta^{2} \rho \mathrm{I} \ddot{\mathrm{v}} " \prime(z, \mathrm{t})-\left(\eta^{2} \rho \mathrm{A}+\rho \mathrm{I}\right) \ddot{\mathrm{v}}(z, \mathrm{t})- \\
& \left(\eta^{2} \mathrm{k}_{\mathrm{w}}+\mathrm{k}_{\mathrm{p}}\right) \mathrm{v}^{\prime \prime}(z, \mathrm{t})+\rho \mathrm{A} \ddot{\mathrm{v}}(z, \mathrm{t})+\mathrm{k}_{\mathrm{w}} \mathrm{v}(z, t)=0
\end{aligned}
$$

where $v(z, \mathrm{t})$ is the transverse displacement, $k_{\mathrm{w}}$ is a Winklertype foundation parameter, $k_{\mathrm{p}}$ is a shear coefficient or a Pasternak- type parameter and $\eta=e_{0}$ a is the small scale effect with $e_{0}$ a constant appropriate to each material and a an is internal characteristic length.

The transverse displacement of the nanotubes can be assumed in the following generalized form:

$$
\mathrm{v}(z, t)=\mathrm{v}(z) \mathrm{e}^{\mathrm{i} \omega \mathrm{t}}
$$

and substituting equation (2) into equation (1) yields:

$$
\begin{aligned}
& \left(\mathrm{EI}+\eta^{2} \mathrm{k}_{\mathrm{p}}-\omega^{2} \eta^{2} \rho \mathrm{I}\right) \mathrm{v}^{\prime \prime \prime}(z)+\omega^{2}\left(\eta^{2} \rho \mathrm{A}+\rho \mathrm{I}\right) \mathrm{v}^{\prime \prime}(z)- \\
& \left(\eta^{2} \mathrm{k}_{\mathrm{w}}+\mathrm{k}_{\mathrm{p}}\right) \mathrm{v}^{\prime \prime}(z)+\left(\mathrm{k}_{\mathrm{w}}-\omega^{2} \rho \mathrm{A}\right) \mathrm{v}(z)=0
\end{aligned}
$$

The boundary conditions are:

$$
\begin{aligned}
& -\left(\mathrm{EI}+\eta^{2} \mathrm{k}_{\mathrm{p}}-\omega^{2} \eta^{2} \rho \mathrm{I}\right) \mathrm{v}^{\prime \prime}(z)+\left(\eta^{2} \mathrm{k}_{\mathrm{w}}+\mathrm{k}_{\mathrm{p}}\right) \mathrm{v}^{\prime}(z)- \\
& \omega^{2}\left(\eta^{2} \rho \mathrm{A}+\rho \mathrm{I}\right) \mathrm{v}^{\prime}(z)-\mathrm{k}_{\mathrm{TL}} \mathrm{v}(z)=0, \quad z=0 \\
& \left(\mathrm{EI}+\eta^{2} \mathrm{k}_{\mathrm{p}}-\omega^{2} \eta^{2} \rho \mathrm{I}\right) \mathrm{v}^{\prime \prime}(z)+\omega^{2} \eta^{2} \rho \mathrm{Av}(z)- \\
& \mathrm{k}_{\mathrm{RL}} \mathrm{v}^{\prime}(z)=0, \quad z=0 \\
& \left(\mathrm{EI}+\eta^{2} \mathrm{k}_{\mathrm{p}}-\omega^{2} \eta^{2} \rho \mathrm{I}\right) \mathrm{v}^{\prime \prime}(z)-\left(\eta^{2} \mathrm{k}_{\mathrm{w}}+\mathrm{k}_{\mathrm{p}}\right) \mathrm{v}^{\prime}(z)+ \\
& \omega^{2}\left(\eta^{2} \rho \mathrm{A}+\rho \mathrm{I}\right) \mathrm{v}^{\prime}(z)-\mathrm{k}_{\mathrm{TR}} \mathrm{v}(z)=0, \quad z=\mathrm{L} \\
& -\left(\mathrm{EI}+\eta^{2} \mathrm{k}_{\mathrm{p}}-\omega^{2} \eta^{2} \rho \mathrm{I}\right) \mathrm{v}^{\prime \prime}(z)-\omega^{2} \eta^{2} \rho \mathrm{Av}(z)- \\
& \mathrm{k}_{\mathrm{RR}} \mathrm{v}^{\prime}(z)=0, \quad z=\mathrm{L}
\end{aligned}
$$

It is convenient to map the physical domain $[0, L]$ on the natural Gaussian domain $[-1,1]$, by means of the following transformation:

$$
\zeta(z)=2 \frac{z}{\mathrm{~L}}-1
$$


where $z$ is the Cartesian co-ordinate and $\zeta$ its natural counterpart. It follows that the differential equation (3) becomes:

$$
\begin{aligned}
& \frac{16}{\mathrm{~L}^{4}}\left(\mathrm{EI}+\eta^{2} \mathrm{k}_{\mathrm{p}}-\omega^{2} \eta^{2} \rho \mathrm{I}\right) \mathrm{v}^{\prime \prime \prime}(\zeta)+\frac{4}{\mathrm{~L}^{2}} \omega^{2}\left(\eta^{2} \rho \mathrm{A}+\rho \mathrm{I}\right) \mathrm{v}^{\prime \prime}(\zeta) \\
& -\frac{4}{\mathrm{~L}^{2}}\left(\eta^{2} \mathrm{k}_{\mathrm{w}}+\mathrm{k}_{\mathrm{p}}\right) \mathrm{v}^{\prime \prime}(\zeta)+\left(\mathrm{k}_{\mathrm{w}}-\omega^{2} \rho \mathrm{A}\right) \mathrm{v}(\zeta)=0
\end{aligned}
$$

dividing by $16 \mathrm{EI} / L^{4}$, the equation (9) becomes:

$$
\begin{aligned}
& \left(1+\eta^{2} \frac{\mathrm{k}_{\mathrm{p}}}{\mathrm{EI}}-\omega^{2} \frac{\eta^{2} \rho \mathrm{I}}{\mathrm{EI}}\right) \mathrm{v}^{\prime \prime \prime}(\zeta)+\frac{\mathrm{L}^{2}}{4 \mathrm{EI}} \omega^{2}\left(\eta^{2} \rho \mathrm{A}+\rho \mathrm{I}\right) \mathrm{v}^{\prime \prime}(\zeta) \\
& -\frac{\mathrm{L}^{2}}{4 \mathrm{EI}}\left(\eta^{2} \mathrm{k}_{\mathrm{w}}+\mathrm{k}_{\mathrm{p}}\right) \mathrm{v}^{\prime \prime}(\zeta)+\frac{\mathrm{L}^{4}}{16 \mathrm{EI}}\left(\mathrm{k}_{\mathrm{w}}-\omega^{2} \rho \mathrm{A}\right) \mathrm{v}(\zeta)=0
\end{aligned}
$$

Assuming the following quantities

$$
\begin{aligned}
& \mathrm{K}_{\mathrm{p} 1}=\frac{\mathrm{k}_{\mathrm{p}} \mathrm{L}^{2}}{4 \mathrm{EI}} ; \mathrm{K}_{\mathrm{p} 2}=\frac{\eta^{2} \mathrm{k}_{\mathrm{p}}}{\mathrm{EI}} ; \mathrm{K}_{\mathrm{w} 1}=\frac{\mathrm{k}_{\mathrm{w}} \mathrm{L}^{4}}{16 \mathrm{EI}} ; \mathrm{K}_{\mathrm{w} 2}=\frac{\eta^{2} \mathrm{k}_{\mathrm{w}} \mathrm{L}^{2}}{4 \mathrm{EI}} ; \\
& \Omega^{2}=\frac{\mathrm{mL}}{16 \mathrm{EI}} ; \Theta^{2}=\frac{\mathrm{mL}^{2} \eta^{2}}{4 \mathrm{EI}} ; \mathrm{I}_{1}=\frac{\rho \mathrm{IL}^{2}}{4 \mathrm{EI}} ; \mathrm{I}_{2}=\frac{\eta^{2} \rho \mathrm{I}}{\mathrm{EI}} .
\end{aligned}
$$

where $m=\rho A$; the equation (10) becomes:

$$
\begin{aligned}
& \left(1+\mathrm{K}_{\mathrm{p} 2}-\omega^{2} \mathrm{I}_{2}\right) \mathrm{v} \mathrm{v}^{\prime \prime \prime}(\zeta)+\omega^{2}\left(\Theta^{2}+\mathrm{I}_{1}\right) \mathrm{v}^{\prime \prime}(\zeta) \\
& -\left(\mathrm{K}_{\mathrm{w} 2}+\mathrm{K}_{\mathrm{p} 1}\right) \mathrm{v}^{\prime \prime}(\zeta)+\left(\mathrm{K}_{\mathrm{w} 1}-\omega^{2} \Omega^{2}\right) \mathrm{v}(\zeta)=0
\end{aligned}
$$
become:

$$
\begin{aligned}
& -\frac{8}{\mathrm{~L}^{3}}\left(\mathrm{EI}+\eta^{2} \mathrm{k}_{\mathrm{p}}-\omega^{2} \eta^{2} \rho \mathrm{I}\right) \mathrm{v}^{\prime \prime \prime}(\zeta)+\frac{2}{\mathrm{~L}}\left(\eta^{2} \mathrm{k}_{\mathrm{w}}+\mathrm{k}_{\mathrm{p}}\right) \mathrm{v}^{\prime}(\zeta)- \\
& \omega^{2}\left(\eta^{2} m+\rho \mathrm{I}\right) \mathrm{v}^{\prime}(\zeta)-\mathrm{k}_{\mathrm{TL}} \mathrm{v}(\zeta)=0, \quad \zeta=-1 \\
& \frac{4}{\mathrm{~L}^{2}}\left(\mathrm{EI}+\eta^{2} \mathrm{k}_{\mathrm{p}}-\omega^{2} \eta^{2} \rho \mathrm{I}\right) \mathrm{v}^{\prime \prime}(\zeta)+\omega^{2} \eta^{2} \rho \mathrm{Av}(\zeta)- \\
& \frac{2}{\mathrm{~L}} \mathrm{k}_{\mathrm{RL}} \mathrm{v}^{\prime}(\zeta)=0, \zeta=-1 \\
& \frac{8}{\mathrm{~L}^{3}}\left(\mathrm{EI}+\eta^{2} \mathrm{k}_{\mathrm{p}}-\omega^{2} \eta^{2} \rho \mathrm{I}\right) \mathrm{v}^{\prime \prime \prime}(\zeta)-\frac{2}{\mathrm{~L}}\left(\eta^{2} \mathrm{k}_{\mathrm{w}}+\mathrm{k}_{\mathrm{p}}\right) \mathrm{v}^{\prime}(\zeta)+ \\
& \frac{2}{\mathrm{~L}} \omega^{2}\left(\eta^{2} m+\rho \mathrm{I}\right) \mathrm{v}^{\prime}(\zeta)-\mathrm{k}_{\mathrm{TR}} \mathrm{v}(\zeta)=0, \zeta=1 \\
& -\frac{4}{\mathrm{~L}^{2}}\left(\mathrm{EI}+\eta^{2} \mathrm{k}_{\mathrm{p}}-\omega^{2} \eta^{2} \rho \mathrm{I}\right) \mathrm{v}^{\prime \prime}(\zeta)-\omega^{2} \eta^{2} \rho \mathrm{Av}(\zeta)- \\
& \frac{2}{\mathrm{~L}} \mathrm{k}_{\mathrm{RR}} \mathrm{v}^{\prime}(\zeta)=0, \quad \zeta=1
\end{aligned}
$$

Dividing equations (13) and (15) by $8 E I / L^{3}$, the equations (14) and (16) by $4 E I / L^{2}$ and taking into account the coefficients of formula (11), one gets:

$$
\begin{aligned}
& \mathrm{C}_{\mathrm{TL}}\left(1+\mathrm{K}_{\mathrm{p} 2}-\omega^{2} \mathrm{I}_{2}\right) \mathrm{v}^{\prime \prime \prime}(\zeta)-\mathrm{C}_{\mathrm{TL}}\left(\mathrm{K}_{\mathrm{w} 2}+\mathrm{K}_{\mathrm{p} 1}\right) \mathrm{v}^{\prime}(\zeta)+ \\
& \omega^{2} \mathrm{C}_{\mathrm{TL}}\left(\Theta^{2}+\mathrm{I}_{1}\right) \mathrm{v}^{\prime}(\zeta)+\mathrm{v}(\zeta)=0, \quad \zeta=-1
\end{aligned}
$$

$$
\begin{aligned}
& -\mathrm{C}_{\mathrm{RL}}\left(1+\mathrm{K}_{\mathrm{p} 2}-\omega^{2} \mathrm{I}_{2}\right) \mathrm{v}^{\prime \prime}(\zeta)-\omega^{2} \Theta^{2} \mathrm{v}(\zeta)+ \\
& \mathrm{v}^{\prime}(\zeta)=0, \quad \zeta=-1 \\
& -\mathrm{C}_{\mathrm{TR}}\left(1+\mathrm{K}_{\mathrm{p} 2}-\omega^{2} \mathrm{I}_{2}\right) \mathrm{v}^{\prime \prime \prime}(\zeta)+C_{\mathrm{TR}}\left(\mathrm{K}_{\mathrm{w} 2}+\mathrm{K}_{\mathrm{p} 1}\right) \mathrm{v}^{\prime}(\zeta)- \\
& \omega^{2} \mathrm{C}_{\mathrm{TR}}\left(\Theta^{2}+\mathrm{I}_{2}\right) \mathrm{v}^{\prime}(\zeta)+\mathrm{v}(\zeta)=0, \quad \zeta=1 \\
& \mathrm{C}_{\mathrm{RR}}\left(1+\mathrm{K}_{\mathrm{p} 2}-\omega^{2} \mathrm{I}_{2}\right) \mathrm{v}^{\prime \prime}(\zeta)+\omega^{2} \Theta^{2} \mathrm{v}(\zeta)+ \\
& \mathrm{v}^{\prime}(\zeta)=0, \quad \zeta=1
\end{aligned}
$$

where the following quantities have been defined:

$$
\mathrm{C}_{\mathrm{TL}}=\frac{8 \mathrm{EI}}{\mathrm{k}_{\mathrm{TL}} \mathrm{L}^{3}} ; \mathrm{C}_{\mathrm{TR}}=\frac{8 \mathrm{EI}}{\mathrm{k}_{\mathrm{TR}} \mathrm{L}^{3}} ; \mathrm{C}_{\mathrm{RL}}=\frac{2 \mathrm{EI}}{\mathrm{k}_{\mathrm{RL}} \mathrm{L}} ; \mathrm{C}_{\mathrm{RR}}=\frac{2 \mathrm{EI}}{\mathrm{k}_{\mathrm{RR}} \mathrm{L}} ;
$$

\section{An overview of Differential Quadrature Method}

In order to discretize the differential equations (12), the natural interval is divided into $n$ segments defined by means of $n+1$ points located at the abscissae $\varsigma_{1}, \varsigma_{2}, \ldots . . \varsigma_{n+1}$. One can assume the set of $n+7$ nodal unknowns:

$$
\mathbf{d}^{\mathrm{T}}=\left\{u_{1}, u_{1}^{\prime}, u_{1}^{\prime \prime}, u_{1}^{\prime \prime \prime}, u_{2}, \ldots, u_{n+1}^{\prime}, u_{n+1}^{\prime \prime}, u_{n+1}^{\prime \prime \prime}\right\}
$$

i.e. the displacement at each nodal points and the first three derivatives at the end points.

Consequently, the displacement $\mathrm{v}(\zeta)$ of the nanotube can be approximated by:

$$
\mathrm{v}(\varsigma)=\boldsymbol{\alpha} \mathbf{C}=\sum_{i=1}^{n+7} \alpha_{i} C_{i}
$$

where $\alpha$ is a row vector of monomials and $\mathbf{C}$ is a column vector of Lagrangian coordinates. One can assign to $\alpha$ the following sequences:

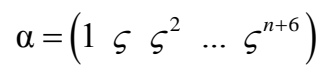

where the nodal points are so defined:

$$
\varsigma_{i}=\frac{2(\mathrm{i}-1)-\mathrm{n}}{\mathrm{n}} ; \quad \mathrm{i}=1,2, \ldots \mathrm{n}+1
$$

Taking the derivative of the equation (23), one gets:

$$
\mathrm{v}^{\prime}(\varsigma)=\boldsymbol{\alpha}^{\prime} \mathbf{C} ; \quad \mathrm{v}^{\prime \prime}(\varsigma)=\boldsymbol{\alpha}^{\prime \prime} \mathbf{C} ; \quad \mathrm{v}^{\prime \prime \prime}(\varsigma)=\boldsymbol{\alpha}^{\prime \prime \prime} \mathbf{C}
$$

and substituting equation (26) into equation (22), following formula is obtained:

$$
\mathbf{d}=\left(\alpha_{1}, \alpha_{1}^{\prime}, \alpha_{1}^{\prime \prime}, \alpha_{1}^{\prime \prime \prime}, \alpha_{2}, \ldots, \alpha_{n+1}, \alpha_{n+1}^{\prime}, \alpha_{n+1}^{\prime \prime}, \alpha_{n+1}^{\prime \prime \prime}\right)^{\mathrm{T}} \mathbf{C}=\mathbf{N}_{0} \mathbf{C}
$$

Following the same approach as in Chen et al. [8], one can define the weighting coefficients of the first four derivatives, as follows:

$$
\mathbf{A}=\mathbf{N}_{0}^{\prime} \mathbf{N}_{0}^{-1}, \quad \mathbf{B}=\mathbf{A} \mathbf{A}, \quad \mathbf{G}=\mathbf{A A A} \mathbf{A}, \quad \mathbf{D}=\mathbf{A} \mathbf{A} \mathbf{A} \mathbf{A}
$$


The differential equation (12) assumes the following form:

$$
\begin{aligned}
& \left(1+\mathrm{K}_{\mathrm{p} 2}\right) \mathrm{v}^{\prime \prime \prime}(\zeta)-\left(\mathrm{K}_{\mathrm{w} 2}+\mathrm{K}_{\mathrm{p} 1}\right) \mathrm{v}^{\prime \prime}(\zeta)+\mathrm{K}_{\mathrm{w} 1} \mathrm{v}(\zeta)= \\
& \omega^{2}\left(\mathrm{I}_{2} v^{\prime \prime \prime \prime}(\zeta)-\left(\Theta^{2}+\mathrm{I}_{1}\right) \mathrm{v}^{\prime \prime}(\zeta)+\Omega^{2} \mathrm{v}(\zeta)\right)
\end{aligned}
$$

The discretized version of the differential equation (29) is:

$$
\left(\begin{array}{cccc}
\mathrm{L}_{11} & \mathrm{~L}_{12} & \cdots & \mathrm{L}_{1, n+7} \\
\mathrm{~L}_{21} & \mathrm{~L}_{22} & \cdots & \mathrm{L}_{2, n+7} \\
\cdots & \cdots & \cdots & \cdots \\
\mathrm{L}_{n+7,1,1} & \mathrm{~L}_{n+7,2} & \mathrm{~L}_{1, n+7} & \mathrm{~L}_{n+7, n+7}
\end{array}\right)=\omega^{2}\left(\begin{array}{cccc}
\mathrm{H}_{11} & \mathrm{H}_{12} & \cdots & \mathrm{H}_{1, n+7} \\
\mathrm{H}_{21} & \mathrm{H}_{22} & \cdots & \mathrm{H}_{2, n+7} \\
\cdots & \cdots & \cdots & \cdots \\
\mathrm{H}_{n+7.1} & \mathrm{H}_{n+7,2.2} & \mathrm{H}_{1, n+7} & \mathrm{H}_{n+7, n+7}
\end{array}\right)
$$

where the matrix $\mathbf{L}$ is the discretized version of the differential operator:

$$
L=\partial^{4} / \partial \zeta^{4}\left(1+\mathrm{K}_{\mathrm{p} 2}\right)-\left(\mathrm{K}_{\mathrm{w} 2}+\mathrm{K}_{\mathrm{p} 1}\right) \partial^{2} / \partial \zeta^{2}+\mathrm{K}_{\mathrm{w} 1}
$$

and $\mathbf{H}$ is given by:

$$
H=\mathrm{I}_{2} \partial^{4} / \partial \zeta^{4}-\left(\Theta^{2}+\mathrm{I}_{1}\right) \partial^{2} / \partial \zeta^{2}+\Omega^{2}
$$

and, as such are given by:

$$
\begin{aligned}
& \mathrm{L}_{\mathrm{i}, \mathrm{j}}=\left(1+\mathrm{K}_{\mathrm{p} 2}\right) \mathrm{D}_{\mathrm{i}, \mathrm{j}}-\left(\mathrm{K}_{\mathrm{w} 2}+\mathrm{K}_{\mathrm{p} 1}\right) \mathrm{B}_{\mathrm{i}, \mathrm{j}}+\mathrm{K}_{\mathrm{w} 1} \delta_{\mathrm{ij}} \\
& \mathrm{H}_{\mathrm{i}, \mathrm{j}}=\mathrm{I}_{2} \mathrm{D}_{\mathrm{i}, \mathrm{j}}-\left(\Theta^{2}+\mathrm{I}_{1}\right) \mathrm{B}_{\mathrm{i}, \mathrm{j}}+\Omega^{2} \delta_{\mathrm{ij}}
\end{aligned}
$$

where $\delta_{\mathrm{ij}}$ is the well-known Kronecker operator. The boundary conditions are:

$$
\begin{aligned}
& \mathrm{C}_{\mathrm{TL}}\left(1+\mathrm{K}_{\mathrm{p} 2}\right) \mathrm{v}_{1}^{\prime \prime \prime}-\mathrm{C}_{\mathrm{TL}}\left(\mathrm{K}_{\mathrm{w} 2}+\mathrm{K}_{\mathrm{p} 1}\right) \mathrm{v}_{1}^{\prime}+\mathrm{v}_{1}= \\
& \omega^{2} \mathrm{C}_{\mathrm{TL}}\left(-\left(\Theta^{2}+\mathrm{I}_{1}\right) \mathrm{v}_{1}^{\prime}+\mathrm{I}_{2} \mathrm{v}_{1}^{\prime \prime \prime}\right) \\
& -\mathrm{C}_{\mathrm{RL}}\left(1+\mathrm{K}_{\mathrm{p} 2}\right) \mathrm{v}_{1}^{\prime \prime}+\mathrm{v}_{1}^{\prime}=-\omega^{2} \mathrm{C}_{\mathrm{RL}} \mathrm{I}_{2} \mathrm{v}_{1}^{\prime \prime}+\omega^{2} \Theta^{2} \mathrm{C}_{\mathrm{RL}} \mathrm{v}_{1} \\
& -\mathrm{C}_{\mathrm{TR}}\left(1+\mathrm{K}_{\mathrm{p} 2}\right) \mathrm{v}_{\mathrm{n}+1}^{\prime \prime \prime}+\mathrm{C}_{\mathrm{TR}}\left(\mathrm{K}_{\mathrm{w} 2}+\mathrm{K}_{\mathrm{p} 1}\right) \mathrm{v}_{\mathrm{n}+1}^{\prime}+\mathrm{v}_{\mathrm{n}+1}= \\
& \omega^{2} \mathrm{C}_{\mathrm{TR}}\left(\left(\Theta^{2}+\mathrm{I}_{1}\right) \mathrm{v}_{\mathrm{n}+1}^{\prime}-\mathrm{I}_{2} \mathrm{v}_{\mathrm{n}+1}^{\prime \prime \prime}\right) \\
& \mathrm{C}_{\mathrm{RR}}\left(1+\mathrm{K}_{\mathrm{p} 2}\right) \mathrm{v}_{\mathrm{n}+1}^{\prime \prime}+\mathrm{v}_{\mathrm{n}+1}^{\prime}=\omega^{2} \mathrm{C}_{\mathrm{RR}} \mathrm{I}_{2} \mathrm{v}_{\mathrm{n}+1}^{\prime \prime}-\omega^{2} \Theta^{2} \mathrm{C}_{\mathrm{RR}} \mathrm{v}_{\mathrm{n}+1}
\end{aligned}
$$

In order to impose the boundary conditions, it is now convenient to interchange the rows (and columns) $(n+4)$ and $(n+5)$ of the matrix $\mathbf{L}$ and $\mathbf{H}$ with the third and fourth rows and (columns) so that the boundary conditions can be immediately imposed. In this way, one obtains a partitioned matrix of the form:

$$
\left(\begin{array}{ll}
\mathbf{L}_{\mathbf{a a}} & \mathbf{L}_{\mathrm{ab}} \\
\mathbf{L}_{\mathbf{b a}} & \mathbf{L}_{\mathbf{b b}}
\end{array}\right)\left(\begin{array}{l}
\mathbf{w}_{\mathrm{c}} \\
\mathbf{w}
\end{array}\right)=\omega^{2}\left(\begin{array}{ll}
\mathbf{H}_{\mathrm{aa}} & \mathbf{H}_{\mathrm{ab}} \\
\mathbf{H}_{\mathrm{ba}} & \mathbf{H}_{\mathrm{bb}}
\end{array}\right)\left(\begin{array}{l}
\mathbf{w}_{\mathrm{c}} \\
\mathbf{w}
\end{array}\right)
$$

and the following eigenvalue problem is obtained:

$$
\begin{aligned}
& \mathbf{L}_{\mathbf{a a}} \mathbf{w}_{c}+\mathbf{L}_{\mathbf{a b}} \mathbf{w}=\omega^{2} \mathbf{H}_{\mathrm{aa}} \mathbf{w}_{c}+\omega^{2} \mathbf{H}_{\mathrm{ab}} \mathbf{w} \\
& \mathbf{L}_{\mathbf{b a}} \mathbf{w}_{c}+\mathbf{L}_{\mathbf{b b}} \mathbf{w}=\omega^{2} \mathbf{H}_{\mathbf{b a}} \mathbf{w}_{c}+\omega^{2} \mathbf{H}_{\mathbf{b b}} \mathbf{w}
\end{aligned}
$$

From the first equation of (39), $\mathbf{w}_{\mathrm{c}}$ which can be singled out so that the second equation becomes

$$
\begin{aligned}
& \left(\mathbf{L}_{\mathrm{ba}}\left(\mathbf{L}_{\mathrm{aa}}-\omega^{2} \mathbf{H}_{\mathrm{aa}}\right)^{-1}\left(\omega^{2} \mathbf{H}_{\mathrm{ab}}-\mathbf{L}_{\mathrm{ab}}\right)+\mathbf{L}_{\mathrm{bb}}\right) \mathbf{w}+ \\
& \left(-\omega^{2} \mathbf{H}_{\mathrm{ba}}\left(\mathbf{L}_{\mathrm{aa}}-\omega^{2} \mathbf{H}_{\mathrm{aa}}\right)^{-1}\left(\omega^{2} \mathbf{H}_{\mathrm{ab}}-\mathbf{L}_{\mathrm{ab}}\right)-\omega^{2} \mathbf{H}_{\mathrm{bb}}\right) \mathbf{w}=0
\end{aligned}
$$

The terms of the equations (39-40) in matrix form are:

$$
\begin{aligned}
& \mathbf{w}_{c}=\left(\begin{array}{llll}
\mathrm{v}_{1}, & \mathrm{v}_{2}^{\prime}, & \mathrm{v}_{\mathrm{n}+1}, & \mathrm{v}_{\mathrm{n}+1}^{\prime}
\end{array}\right)^{\mathrm{T}} ; \\
& \mathbf{w}=\left(\begin{array}{llllll}
\mathrm{v}_{2}, & \cdots & \mathrm{v}_{1}^{\prime \prime}, & \mathrm{v}_{1}^{\prime \prime \prime}, & \mathrm{v}_{\mathrm{n}+1}^{\prime \prime}, & \mathrm{v}_{\mathrm{n}+1}^{\prime \prime \prime}
\end{array}\right)^{\mathrm{T}} . \\
& \mathbf{L}_{\mathrm{aa}}(1,2)=-\mathrm{C}_{\mathrm{TL}}\left(\mathrm{K}_{\mathrm{w} 2}+\mathrm{K}_{\mathrm{p} 1}\right) ; \mathbf{L}_{\mathrm{aa}}(3,4)=\mathrm{C}_{\mathrm{TR}}\left(\mathrm{K}_{\mathrm{w} 2}+\mathrm{K}_{\mathrm{p} 1}\right) \text {. }
\end{aligned}
$$

$\mathbf{L}_{\mathbf{b a}}=\left(\begin{array}{cccc}L_{5,1} & L_{5,2} & L_{5, \mathrm{n}+4} & L_{5, \mathrm{n}+5} \\ \cdots & \cdots & \cdots & \cdots \\ L_{3,1} & L_{3,2} & L_{3, \mathrm{n}+4} & L_{3, \mathrm{n}+5} \\ L_{4,1} & L_{4,2} & L_{4, \mathrm{n}+4} & L_{4, \mathrm{n}+5} \\ L_{\mathrm{n}+6,1} & L_{\mathrm{n}+6,2} & L_{\mathrm{n}+6, \mathrm{n}+4} & L_{\mathrm{n}+6, \mathrm{n}+5} \\ L_{\mathrm{n}+7,1} & L_{\mathrm{n}+7,2} & L_{\mathrm{n}+7, \mathrm{n}+4} & L_{\mathrm{n}+7, \mathrm{n}+5}\end{array}\right)$

$$
\mathbf{L}_{\mathbf{b b}}=\left(\begin{array}{cccccc}
L_{5,5} & \vdots & L_{5,3} & L_{5,4} & L_{5, \mathrm{n}+6} & L_{5, \mathrm{n}+7} \\
\cdots & \vdots & \cdots & \cdots & \cdots & \cdots \\
L_{3,5} & \vdots & L_{3,3} & L_{3,4} & L_{3, \mathrm{n}+6} & L_{3, \mathrm{n}+7} \\
L_{4,5} & \vdots & L_{4,3} & L_{4,4} & L_{4, \mathrm{n}+6} & L_{4, \mathrm{n}+7} \\
L_{\mathrm{n}+6,5} & \vdots & L_{\mathrm{n}+6,3} & L_{\mathrm{n}+6,4} & L_{\mathrm{n}+6, \mathrm{n}+6} & L_{\mathrm{n}+6, \mathrm{n}+7} \\
L_{\mathrm{n}+7,5} & \vdots & L_{\mathrm{n}+7,3} & L_{\mathrm{n}+7,4} & L_{\mathrm{n}+7, \mathrm{n}+6} & L_{\mathrm{n}+7, \mathrm{n}+7}
\end{array}\right)
$$

$\mathbf{H}_{\text {aa }}(1,2)=-\mathrm{C}_{\mathrm{TL}}\left(\Theta^{2}+\mathrm{I}_{1}\right) ; \mathbf{H}_{\mathrm{aa}}(3,4)=\mathrm{C}_{\mathrm{TR}}\left(\Theta^{2}+\mathrm{I}_{1}\right)$

$\mathbf{H}_{\text {aa }}(2,1)=\Theta^{2} \mathrm{C}_{\mathrm{RL}} ; \mathbf{H}_{\mathrm{aa}}(4,3)=-\Theta^{2} \mathrm{C}_{\mathrm{RR}}$

$\mathbf{H}_{\mathrm{ab}}(1, \mathrm{n}+5)=\mathrm{C}_{\mathrm{TL}} \mathrm{I}_{2} ; \mathbf{H}_{\mathrm{ab}}(2, \mathrm{n}+4)=-\mathrm{C}_{\mathrm{RL}} \mathrm{I}_{2} ;$

$\mathbf{H}_{\mathrm{ab}}(3, \mathrm{n}+7)=-\mathrm{C}_{\mathrm{TR}} \mathrm{I}_{2} ; \mathbf{H}_{\mathrm{ab}}(4, \mathrm{n}+6)=\mathrm{C}_{\mathrm{RR}} \mathrm{I}_{2}$.

$\mathbf{H}_{\mathrm{ba}}=\left(\begin{array}{cccc}H_{5,1} & H_{5,2} & H_{5, \mathrm{n}+4} & H_{5, \mathrm{n}+5} \\ \cdots & \cdots & \cdots & \cdots \\ H_{3,1} & H_{3,2} & H_{3, \mathrm{n}+4} & H_{3, \mathrm{n}+5} \\ H_{4,1} & H_{4,2} & H_{4, \mathrm{n}+4} & H_{4, \mathrm{n}+5} \\ H_{\mathrm{n}+6,1} & H_{\mathrm{n}+6,2} & H_{\mathrm{n}+6, \mathrm{n}+4} & H_{\mathrm{n}+6, \mathrm{n}+5} \\ H_{\mathrm{n}+7,1} & H_{\mathrm{n}+7,2} & H_{\mathrm{n}+7, \mathrm{n}+4} & H_{\mathrm{n}+7, \mathrm{n}+5}\end{array}\right)$ 


$$
\mathbf{H}_{\mathbf{b b}}=\left(\begin{array}{cccccc}
H_{5,5} & \vdots & H_{5,3} & H_{5,4} & H_{5, \mathrm{n}+6} & H_{5, \mathrm{n}+7} \\
\cdots & \vdots & \cdots & \cdots & \cdots & \cdots \\
H_{3,5} & \vdots & H_{3,3} & H_{3,4} & H_{3, \mathrm{n}+6} & H_{3, \mathrm{n}+7} \\
H_{4,5} & \vdots & H_{4,3} & H_{4,4} & H_{4, \mathrm{n}+6} & H_{4, \mathrm{n}+7} \\
H_{\mathrm{n}+6,5} & \vdots & H_{\mathrm{n}+6,3} & H_{\mathrm{n}+6,4} & H_{\mathrm{n}+6, \mathrm{n}+6} & H_{\mathrm{n}+6, \mathrm{n}+7} \\
H_{\mathrm{n}+7,5} & \vdots & H_{\mathrm{n}+7,3} & H_{\mathrm{n}+7,4} & H_{\mathrm{n}+7, \mathrm{n}+6} & H_{\mathrm{n}+7, \mathrm{n}+7}
\end{array}\right)
$$

\section{Numerical examples}

Let us consider a single-walled nanotube having the same geometrical and physical properties used in Kiani [5]: $\mathrm{R}=3 \mathrm{~nm}$ in mean radius, $\mathrm{t}=0.34 \mathrm{~nm}$ wall thickness, $\mathrm{E}=1 \mathrm{TPa}$, Young's modulus and mass density $\rho=2.5 \times 10^{3} \mathrm{~kg} / \mathrm{m}^{3}$. Moreover, the non dimensional coefficient $\lambda=\mathrm{L} / \mathrm{r}_{\mathrm{b}}$, with $\mathrm{r}_{\mathrm{b}}=(\mathrm{I} / \mathrm{A})^{1 / 2}$, is assumed.

Table 1: Simply supported-simply supported SWCNT

\begin{tabular}{|c|c|c|c|c|c|c|}
\hline \multirow{2}{*}{$\lambda$} & \multicolumn{3}{|c|}{$\eta=0.1$} & \multicolumn{3}{c|}{$\eta=0.3$} \\
\cline { 2 - 7 } & NEBT & {$[15]$} & DQM & NEBT & {$[15]$} & DQM \\
\hline \multirow{4}{*}{10} & 3.0006 & 2.9972 & 2.9185 & 2.6208 & 2.6177 & 2.6177 \\
\cline { 2 - 7 } & 5.3234 & 5.3202 & 5.3202 & 3.9601 & 3.9580 & 3.9580 \\
\cline { 2 - 7 } & 6.8659 & 6.8587 & 6.8583 & 4.6463 & 4.6426 & 4.6424 \\
\cline { 2 - 7 } & 7.8384 & 7.8248 & 7.8434 & 5.0270 & 5.0210 & 5.0290 \\
\hline \multirow{4}{*}{30} & 8.4568 & 8.4356 & 8.6382 & 5.2530 & 5.2446 & 5.3129 \\
\hline & 3.0638 & 3.0602 & 3.0602 & 2.6413 & 2.6727 & 2.6727 \\
\cline { 2 - 7 } & 5.7237 & 5.7199 & 5.7200 & 4.0803 & 4.2554 & 4.2554 \\
\cline { 2 - 7 } & 7.8633 & 7.8530 & 7.8525 & 4.9058 & 5.3157 & 5.3153 \\
\hline & 9.5478 & 9.5233 & 9.5570 & 5.4266 & 6.1110 & 6.1305 \\
\hline & 10.8820 & 10.8346 & 10.3530 & 5.7775 & 6.7361 & 6.9669 \\
\hline
\end{tabular}

In Table 1, the first five non dimensional free frequencies $\Lambda_{\mathrm{i}}=\left(\omega_{\mathrm{i}}^{2} \times \rho \mathrm{AL} / \mathrm{EI}\right)^{1 / 4}$ of simply supported-simply supported SWCNT are reported, for varying values of the non dimensional coefficient $\lambda=(10,30)$ and of the parameter $\eta=$ $(0.1,0.3)$ while the elastic medium parameters are assumed to be zero. The results have been calculated using a general code, developed in Mathematica [14]. A numerical comparison, illustrated in Table 1, is furnished between the results given by the proposed method (DQM) and the results given by Kiani $[5,15]$ by means NEBT method. As one can see there is a good agreement between the two numerical approaches.

In Figure 1, the free frequencies of clamped-clamped SWCNT, having the same geometric and mechanical properties of the above example, are reported. Fixed $\lambda=10$ and $\eta=0.3$, six curves have been plotted for different values of Winkler-type parameter $\mathrm{K}_{\mathrm{wl}}=[0,1,10,50,100]$ of equation (11). On the axis $x$ the values of $K_{\mathrm{pl}}$, (see equation 11) are reported, while on the axis y the $\Lambda_{1}$ values are listed. As can be seen, the fundamental frequencies increase the Pasternak parameter and Winkler coefficients.

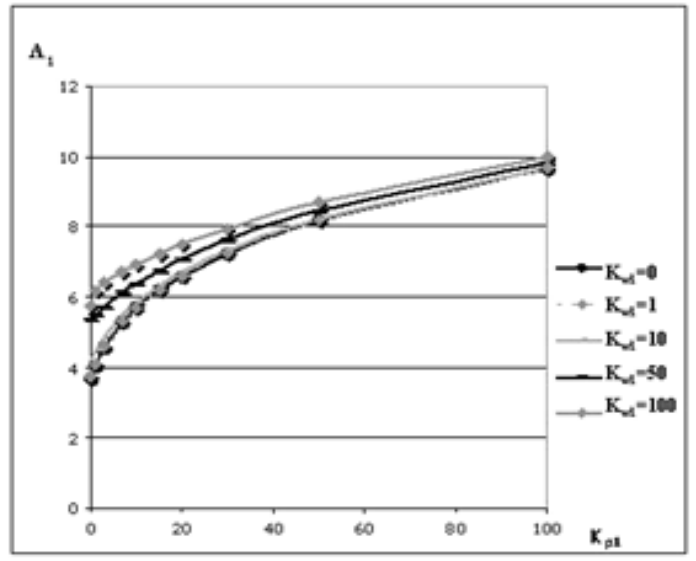

Fig.1: Clamped-Clamped SWCNT

\section{References}

[1] C.Q. Ru, "Column buckling of multiwalled carbon nanotubes with interlayer radial displacements", Phys. Rev. B, Vol. 62, no 4, pp. 16962-16967, 2000.

[2] [Q. Wang, T. Hu, and Q.J. Chen, "Bending instability characteristics of double walled nanotubes", Phys. Rev. B, Vol. 71, no 4, pp. 045403 045412, 2005.

[3] R. F. Gibson, E. O. Ayorinde, and Y.F. Wen, "Vibrations of carbon nanotubes on their composites: a review", Comp. Science and Techn., Vol. 67, pp. 1-28, 2007.

[4] S. Adali, "Variational principles for transversely vibrating multiwalled carbon nanotubes based on nonlocal Euler-Bernoulli beam model", Nanoletters, 9, pp. 1737-41, 2009.

[5] K. Kiani, "A meshless approach for free transverse vibration of embedded single-walled nanotubes with arbitrary boundary conditions accounting for nonlocal effect", Int. J. Mech. Sci., Vol. 52, pp. 13431356, 2010.

[6] R. E. Bellman and J. Casti, "Differential quadrature and long-term integration", J. Math. Anal. Appl., Vol. 34, pp. 235-238, 1971.

[7] C. W. Bert and M. Malik, "Differential quadrature method in computational mechanics", Appl. Mech. Rev., Vol. 49, no. 1, pp. 1-28, 1996.

[8] W. Chen, A. G. Striz, and C. W. Bert, "A new approach to the differential quadrature method for fourth-order equations," Int. J. Num. Meth. Engng., Vol. 40, pp. 1941-1956, 1997.

[9] M. A. De Rosa and C. Franciosi, "On natural boundary conditions and DQM", Mech. Res. Comm., Vol. 25(3), pp. 279-286, 1998.

[10] M. A. De Rosa and C. Franciosi, "Non-classical boundary conditions and DQM", J. of Sound and Vibr., Vol. 212(4), pp. 743-748, 1998.

[11] M. A. De Rosa and M. Lippiello, "Non-classical boundary conditions and DQM four double beams", Mech. Res. Comm., Vol. 34 no 7-8, pp. 538-544, 2007.

[12] M. A. De Rosa, N. M. Auciello, and M. Lippiello, "Dynamic stability analysis and DQM for beams with variable cross-section", Mech. Res. Comm., Vol. 35 no 3, pp. 187-192, 2008.

[13] M. A. De Rosa, M. Lippiello, M. J. Maurizi, and H.D. Martin, "El método de cuadratura diferencial en el análisis dinámico del sistema doble-viga, simplemente apoyado y de sección circular variable", Primer Congreso Argentino De Ingenieria Mecanica, I CAIM 2008, 1-3 Octubre 2008. La Piedad Ed., Bahia Blanca, Argentina 2008.

[14] S. Wolfram, The Mathematica 8. Wolfram Media Cambridge: Cambridge University Press; 2010.

[15] K. Kiani, B. Mehri, "Assessment of nanotube structures under a moving nanoparticle using nonlocal beam theories.", J. of Sound and Vibr., Vol. 329, no 11, pp. 2241-2264, 2010. 